\title{
Evaluation of Acceleration and Jerk Models in Radar and IRST Data Fusion for Tracking Evasive Maneuvering Target
}

\author{
VPS Naidu \\ Multi Sensor Data Fusion Lab, National Aerospace Laboratories, Bangalore, India
}

\begin{abstract}
In general, the motion of maneuvering targets is described by acceleration model which is a kinematic model including position derivatives up to the second order. It is found that for tracking highly maneuvering targets, it is necessary to include higher order position derivatives in the model. A model including derivatives of position up to the third order called the jerk model is presented. In this paper, the tracking performance of an extended Kalman filter with the jerk model is evaluated and compared with that of an extended Kalman filter with acceleration model for fusion and tracking of a maneuvering target sensed by IRST and radar.
\end{abstract}

\section{Nomenclature}

$\begin{array}{ll}E K F & =\text { extended Kalman filter } \\ I R S T & =\text { infrared search and track } \\ M S D F & =\text { multi sensor data fusion } \\ X & =\text { state vector } \\ r(k) & =\text { range at time index } k \\ \theta(k) & =\text { azimuth at time index } k \\ \varphi(k) & =\text { elevation at time index } k \\ E\{.\} & =\text { mathematical expectation } \\ \alpha & =\text { reciprocal of the jerk time constant } \\ \delta(\tau) & =\text { unit impulse function } \\ Q & =\text { noise covariance matrix } \\ \Phi & =\text { state transition matrix } \\ T & =\text { sampling time }\end{array}$

\section{Introduction}

$\mathrm{K}$ ALMAN filters are extensively used in target tracking applications ${ }^{1}$. In these applications, the state of the target includes its position and the time-derivatives of the position. For targets moving with constant velocity, these models include the first derivative of position and for targets moving with constant acceleration they include second derivative of position ${ }^{2}$. Models with second order derivatives are preferred for tracking maneuvering targets and referred to as acceleration models. However, for highly maneuvering targets, it is seen ${ }^{1}$ that the tracking performance of acceleration model is not very accurate. The reason for this degradation could be attributed to the fact that under large maneuver conditions, higher order derivatives of position become significant. In ref. [1] an approach to handle highly maneuvering targets is presented when the target executes large maneuvers. The procedure involves inclusion of third order derivative of the target position in the state model and this model is termed 'jerk model' for target tracking.

In the Singer model ${ }^{3}$, which is the standard model for modeling target maneuvers, the target acceleration is assumed to be first-order Markov process. The same concept has been extended to model target jerk. In this paper, the jerk is modeled as a zero-mean first order Markov process and a four state extended Kalman filter (EKF) employing jerk model is evolved. Detailed derivations of most of the expressions in ref. [1] are provided. IRST and Radar mathematical models are presented and used to simulated the data. The performance of jerk model is compared with constant acceleration model in Radar and IRST data fusion using simulated trajectory of a target undergoing evasive maneuver. Results are presented in terms of estimation errors in state variables, root mean 
square error in position, velocity and acceleration, percentage fit error in $\mathrm{x}$-, $\mathrm{y}$ - and z-positions, and normalized estimation error square, normalized innovation square and autocorrelation of innovation sequences.

\section{Sensor Models}

In most of the aerospace applications sensors are needed in order to localize the position of the targets, track the target as they move. Since estimation is to be done with the aid of sensors, appropriate sensors are chosen to collect data for different applications. Sensor mathematical models are required for generating realistic sensor data for validating estimation and tracking algorithms which could also be used for Multi Sensor Data Fusion (MSDF) applications. The estimation and tracking algorithms for real time applications need to be validated using simulation studies. The mathematical models of the sensors help in generating realistic data. Radar and IRST measurements are expressed in spherical coordinates.

Radar measurement model:

$$
\begin{array}{lll}
\text { range: } & r_{m}^{R}(k)=r(k)+v_{r}^{R}(k) \quad(\mathrm{m}) \\
\text { azimuth: } & \theta_{m}^{R}(k)=\theta(k)+v_{\theta}^{R}(k) \quad(\mathrm{rad}) \\
\text { elevation: } & \varphi_{m}^{R}(k)=\varphi(k)+v_{\varphi}^{R}(k) \quad(\mathrm{rad})
\end{array}
$$

where $v_{r}^{R}(k), v_{\theta}^{R}(k)$ and $v_{\varphi}^{R}(k)$ are zero-mean white noise sequence with standard deviations $\sigma_{r}^{R}, \sigma_{\theta}^{R}$ and $\sigma_{\varphi}^{R}$ respectively

IRST measurement model:

$$
\begin{array}{ll}
\text { azimuth: } \quad \theta_{m}^{I}(k)=\theta(k)+v_{\theta}^{I}(k) \quad(\mathrm{rad}) \\
\text { elevation: } \varphi_{m}^{I}(k)=\varphi(k)+v_{\varphi}^{I}(k) \quad(\mathrm{rad})
\end{array}
$$

where $v_{\theta}^{I}(k)$ and $v_{\varphi}^{I}(k)$ are zero-mean white noise sequence with standard deviations $\sigma_{\theta}^{I}$ and $\sigma_{\varphi}^{I}$ respectively

Superscript $R$ and $I$ are used to indicate the radar and IRST measurements. The index $k$ indicates the sample/scan number. It is assumed that both the sensors are measuring the same target denoted by $(r(k), \theta(k), \varphi(k))$. The subscript $m$ indicates measurement and $v$ is the measurement noise added to simulate the realistic measurements.

\section{Target Motion Modeling with Jerk}

Target jerk could be described as a process that is correlated in time. Fast and evasive maneuvers will result in faster decay of jerk correlation compared to slow and lazy maneuvers, which will have longer correlation times. Target jerk can be represented using exponential correlation function as:

$$
r_{j}(\tau)=E\{j(t) j(t+\tau)\}=\sigma_{j}^{2} e^{-\alpha|\tau|}
$$

where $\sigma_{j}^{2}$ - variance of the target jerk

$$
\begin{aligned}
& \alpha \text { - reciprocal of the jerk time constant } \tau \\
& E\{.\} \text { - mathematical expectation } \\
& j(t) \text {-jerk at time } t
\end{aligned}
$$

The Laplace transform of the jerk correlation function $r_{j}(\tau)$ is:

$$
\begin{aligned}
& R(s)=L\left\{r_{j}(\tau)\right\}=\int_{-\infty}^{\infty} e^{-s \tau} \sigma_{j}^{2} e^{-\alpha|\tau|} d \tau=\sigma_{J}^{2}\left[\int_{-\infty}^{0} e^{-s \tau} e^{\alpha \tau} d \tau+\int_{0}^{\infty} e^{-s \tau} e^{-\alpha \tau} d \tau\right] \\
& =\sigma_{j}^{2}\left[\int_{-\infty}^{0} e^{(-s+\alpha) \tau} d \tau+\int_{0}^{\infty} e^{-(s+\alpha) \tau} d \tau\right]=\sigma_{j}^{2}\left[\left.\frac{e^{(-s+\alpha) \tau}}{-s+\alpha}\right|_{-\infty} ^{0}+\left.\frac{e^{-(s+\alpha) \tau}}{-(s+\alpha)}\right|_{0} ^{\infty}\right]
\end{aligned}
$$




$$
\begin{aligned}
& =\sigma_{j}^{2}\left[\frac{1}{-s+\alpha}-0+0-\frac{1}{-(s+\alpha)}\right]=\sigma_{j}^{2}\left[\frac{-1}{s-\alpha}+\frac{1}{s+\alpha}\right] \\
& =\frac{-2 \alpha \sigma_{j}^{2}}{(s-\alpha)(s+\alpha)}=H(s) H(-s) W(s) \\
& \text { where } H(s)=\frac{1}{(s+\alpha)} \\
& \text { and } W(s)=2 \alpha \sigma_{j}^{2}
\end{aligned}
$$

The term $H(s)$ is the transfer function of the whitening filter for the jerk $j(t)$ and $W(s)$ is the transform of the white noise $w(t)$ that drives $j(t)$. From the transfer function of eq. (5), the differential equation for jerk can be written as:

$$
\dot{j}(t)=-\alpha \quad j(t)+w(t)
$$

The autocorrelation function of the white noise is computed using inverse Laplace transform of $\mathrm{W}(\mathrm{s})$ :

$$
r_{w}(\tau)=L^{-1}\left\{2 \alpha \sigma_{j}^{2}\right\}=2 \alpha \sigma_{j}^{2} \delta(\tau)=Q_{w} \delta(\tau)
$$

where $\delta(\tau)$ is the unit impulse function or "delta" function located at the origin

$$
\text { and } Q_{w}=2 \alpha \sigma_{j}^{2} \text { is the variance of } w(t)
$$

The correlation parameter $\alpha$ in eq. (7) is used to model different classes of targets. Small values of $\alpha$ are used for targets with sustained jerk levels and high $\alpha$ values for targets with rapidly fluctuating jerk. The white noise component in eq. (7) helps superimpose random jerk components over sustained jerk component. Effectively $\alpha$ helps to adjust the model order to fractional values with low $\alpha$ representing jerk model and high $\alpha$ corresponding to acceleration model. Using eq. (7), the state space form for the continuous-time jerk model can be written as

$$
\frac{d}{d t}\left[\begin{array}{c}
x \\
\dot{x} \\
\ddot{x} \\
\dddot{x}
\end{array}\right]=\left[\begin{array}{cccc}
0 & 1 & 0 & 0 \\
0 & 0 & 1 & 0 \\
0 & 0 & 0 & 1 \\
0 & 0 & 0 & -\alpha
\end{array}\right]\left[\begin{array}{c}
x \\
\dot{x} \\
\ddot{x} \\
\dddot{x}
\end{array}\right]+\left[\begin{array}{l}
0 \\
0 \\
0 \\
1
\end{array}\right] w(t)
$$

where $x, \dot{x}, \ddot{x}, \dddot{x}$ denote the position, velocity, acceleration and jerk of the target respectively. Eq.(9) has the standard form:

$$
\dot{X}=A X+B w(t)
$$

The measurement vector at $(k+1)^{\text {th }}$ instant is:

$$
Z(k+1)=H X(k+1)+v(k+1)
$$

where $H$ : Observation matrix

$v$ : measurement noise vector assumed uncorrelated to the process noise.

\section{Tracking in One Dimension using Jerk Model}

In this section, detailed derivations are provided for the state model and process noise covariance matrix for a target moving in one dimension with constant jerk. The same can be extended for target tracking in three dimensions. Discretization of eq.(9) results in

$$
\begin{aligned}
& \qquad X(k+1)=\Phi(k+1, k) X(k)+u(k) \\
& \text { where } \Phi(k+1, k)=e^{A\left(t_{k+1}-t_{k}\right)}
\end{aligned}
$$

is a transition matrix of the linear time-invariant system between the instants $k$ and $k+1$, and $u(k)$ is discrete white noise vector defined as: 


$$
u(k)=\int_{t_{k}}^{t_{k+1}} \Phi\left(t_{k+1}, \tau\right) B(\tau) w(\tau) d(\tau)
$$

The state transition matrix can be obtained from (eq. 13) by series expansion of the right-hand side as ${ }^{5}$

$$
\Phi(T)=\left[\begin{array}{cccc}
1 & T & \frac{T^{2}}{2} & p \\
0 & 1 & T & q \\
0 & 0 & 1 & r \\
0 & 0 & 0 & s
\end{array}\right]
$$

where $T=t_{k+1}-t_{k}$

$$
\begin{aligned}
& p=\frac{T^{3}}{3 !}-\frac{\alpha T^{4}}{4 !}+\frac{\alpha^{2} T^{5}}{5 !}-\ldots=\frac{2-2 \alpha T+\alpha^{2} T^{2}-2 e^{-\alpha T}}{2 \alpha^{3}} \\
& q=\frac{T^{2}}{2 !}-\frac{\alpha T^{3}}{3 !}+\frac{\alpha^{2} T^{4}}{4 !}-\ldots=\frac{e^{-\alpha T}-1+\alpha T}{\alpha^{2}} \\
& r=T-\frac{\alpha T^{2}}{2 !}+\frac{\alpha^{2} T^{3}}{3 !}-\ldots=\frac{1-e^{-\alpha T}}{\alpha} \\
& s=1-\alpha T+\frac{\alpha T^{2}}{2 !}-\frac{\alpha^{2} T^{3}}{3 !}+\ldots=e^{-\alpha T}
\end{aligned}
$$

For small $\alpha T$ the state transition matrix can be written as:

$$
\lim _{\alpha \rightarrow 0} \Phi(T)=\left[\begin{array}{cccc}
1 & T & \frac{T^{2}}{2} & \frac{T^{3}}{6} \\
0 & 1 & T & \frac{T^{2}}{2} \\
0 & 0 & 1 & T \\
0 & 0 & 0 & 1
\end{array}\right]
$$

which means that the filter assumes the jerk to be constant between the sampling instants.

The uncertainty in state estimation due to random target dynamics or miss-modeling of target dynamics is typically represented by the process noise covariance matrix $Q^{1}$. The variance $Q(k)$ of the process noise $u(k)$ (eq.14) is given by

$$
\begin{aligned}
Q(k)= & E\left\{u(k) u^{T}(k)\right\} \\
& =\int_{t_{k}}^{t_{k+1}} \Phi\left(t_{k+1}, \tau\right) B(\tau) w(\tau) d \tau \int_{t_{k}}^{t_{k+1}} w^{T}(v) B^{T}(v) \Phi^{T}\left(t_{k+1}, v\right) d v \\
& =\int_{t_{k}}^{t_{k+1}} \int_{t_{k}}^{t_{k+1}} \Phi\left(t_{k+1}, \tau\right) B(\tau) E\left\{w(\tau) w^{T}(v)\right\} B^{T}(v) \Phi^{T}\left(t_{k+1}, v\right) d \tau d v
\end{aligned}
$$

Here $E\left\{w(\tau) w^{T}(v)\right\}=Q_{w} \delta(\tau)$ and $\Phi\left(t_{k+1}, \tau\right)=\Phi\left(t_{k+1}-\tau\right)$ for time-invariant systems. Hence eq. (18) can be written as:

$$
Q(k)=Q_{w} \int_{t_{k}}^{t_{k+1}} \Phi\left(t_{k+1}-\tau\right) B(\tau) B^{T}(\tau) \Phi^{T}\left(t_{k+1}-\tau\right) d \tau
$$

Using expanded form of B and matrix $\Phi$ from eq. (15) $Q(k)$ can be written as 


$$
Q(k)=Q_{w}\left[\begin{array}{cccc}
\int p^{2} d \tau & \int p q d \tau & \int p r d \tau & \int p s d \tau \\
\int q p d \tau & \int q^{2} d \tau & \int q r d \tau & \int q s d \tau \\
\int r p d \tau & \int r q d \tau & \int r^{2} d \tau & \int r s d \tau \\
\int s p d \tau & \int s q d \tau & \int s r d \tau & \int s^{2} d \tau
\end{array}\right]=2 \alpha \sigma_{j}^{2}\left[\begin{array}{cccc}
q_{11} & q_{12} & q_{13} & q_{14} \\
q_{21} & q_{22} & q_{23} & q_{24} \\
q_{31} & q_{32} & q_{33} & q_{34} \\
q_{41} & q_{42} & q_{43} & q_{44}
\end{array}\right]
$$

Here the quantities $p, q, r$ and $s$ are evaluated at the instant $\left(t_{k+1}-\tau\right)$. The elements in the matrix are computed as:

$$
q_{24}=\int_{t_{k}}^{t_{k+1}} q\left(t_{k+1}-u\right) s\left(t_{k+1}-u\right) d u
$$

For simplicity if we assume that $t_{k+1}=t$, then $t_{k}=t-T$. The eq.(20) can be written as:

$$
\begin{aligned}
q_{24} & =\int_{t-T}^{t} q(t-u) s(t-u) d u \\
& =\int_{t-T}^{t} \frac{e^{-\alpha(t-u)}-1+\alpha(t-u)}{\alpha^{2}} e^{-\alpha(t-u)} d u=\frac{1}{\alpha^{2}} \int_{t-T}^{t} e^{-2 \alpha(t-u)}-e^{-\alpha(t-u)}+\alpha(t-u) e^{-\alpha(t-u)} d u \\
& =\frac{1}{\alpha^{2}}\left[\frac{e^{-2 \alpha(t-u)}}{2 \alpha}-\frac{e^{-\alpha(t-u)}}{\alpha}+\frac{\alpha t e^{-\alpha(t-u)}}{\alpha}-\frac{\alpha u e^{-\alpha(t-u)}}{\alpha}+\frac{\alpha e^{-\alpha(t-u)}}{\alpha^{2}}\right]_{t-T}^{t} \\
& =\frac{1}{\alpha^{2}}\left[\frac{e^{-2 \alpha(t-u)}}{2 \alpha}+\frac{\alpha(t-u) e^{-\alpha(t-u)}}{\alpha}\right]_{t-T}^{t}=\frac{1}{2 \alpha^{3}}\left[e^{-2 \alpha(t-u)}+2 \alpha(t-u) e^{-\alpha(t-u)}\right]_{t-T}^{t} \\
& =\frac{1}{2 \alpha^{3}}\left[e^{-2 \alpha(t-t)}+2 \alpha(t-t) e^{-\alpha(t-t)}-e^{-2 \alpha(t-t+T)}-2 \alpha(t-t+T) e^{-\alpha(t-t+T)}\right] \\
& =\frac{1}{2 \alpha^{3}}\left(1-e^{-2 \alpha T}-2 \alpha T e^{-\alpha T}\right)
\end{aligned}
$$

Similarly the other elements in the matrix can be computed and are given below:

$$
\begin{aligned}
q_{11} & =\int_{t-T}^{t} p^{2}(t-u) d u \\
& =\frac{1}{2 \alpha^{7}}\left(\frac{\alpha^{5} T^{5}}{10}-\frac{\alpha^{4} T^{4}}{2}+\frac{4 \alpha^{3} T^{3}}{3}-2 \alpha^{2} T^{2}+2 \alpha T-3+4 e^{-\alpha T}+2 \alpha^{2} T^{2} e^{-\alpha T}-e^{-2 \alpha T}\right) \\
q_{12} & =\int_{t-T}^{t} p(t-u) q(t-u) d u \\
& =\frac{1}{2 \alpha^{6}}\left(1-2 \alpha T+2 \alpha^{2} T^{2}-\alpha^{3} T^{3}+\frac{\alpha^{4} T^{4}}{4}+e^{-2 \alpha T}+2 \alpha T e^{-\alpha T}-2 e^{-\alpha T}-\alpha^{2} T^{2} e^{-\alpha T}\right) \\
q_{21} & =q_{12} \\
q_{13} & =q_{31}=\int_{t-T}^{t} p(t-u) r(t-u) d u
\end{aligned}
$$




$$
\begin{gathered}
=\frac{1}{2 \alpha^{5}}\left(2 \alpha T-\alpha^{2} T^{2}+\frac{\alpha^{3} T^{3}}{3}-3-e^{-2 \alpha T}+4 e^{-\alpha T}+\alpha^{2} T^{2} e^{-\alpha T}\right) \\
q_{14}=q_{41}=\int_{t-T}^{t} p(t-u) s(t-u) d u=\frac{1}{2 \alpha^{43}}\left(1+e^{-2 \alpha T}-2 e^{-\alpha T}-\alpha^{2} T^{2} e^{-\alpha T}\right) \\
q_{22}=\int_{t-T}^{t} q^{2}(t-u) d u=\frac{1}{2 \alpha^{5}}\left(1-e^{-2 \alpha T}+\frac{2 \alpha^{3} T^{3}}{3}+2 \alpha T-2 \alpha^{2} T^{2}-4 \alpha T e^{-\alpha T}\right) \\
q_{23}=q_{32}=\int_{t-T}^{t} q(t-u) r(t-u) d u=\frac{1}{2 \alpha^{4}}\left(1+\alpha^{2} T^{2}-2 \alpha T+2 \alpha T e^{-\alpha T}+e^{-2 \alpha T}-2 e^{-\alpha T}\right) \\
q_{33}=\int_{t-T}^{t} r^{2}(t-u) d u=\frac{1}{2 \alpha^{3}}\left(4 e^{-\alpha T}-e^{-2 \alpha T}+2 \alpha T-3\right) \\
q_{34}=q_{43}=\int_{t-T}^{t} r(t-u) s(t-u) d u=\frac{1}{2 \alpha^{2}}\left(1-2 e^{-\alpha T}+e^{-2 \alpha T}\right) \\
q_{44}=\int_{t-T}^{t} s^{2}(t-u) d u=\frac{1}{2 \alpha}\left(1-e^{-2 \alpha T}\right)
\end{gathered}
$$

For small $\alpha T$ value the process noise covariance matrix becomes

$$
Q(k)=2 \alpha \sigma_{j}^{2}\left[\begin{array}{cccc}
\frac{T^{7}}{252} & \frac{T^{6}}{72} & \frac{T^{5}}{30} & \frac{T^{4}}{24} \\
\frac{T^{6}}{72} & \frac{T^{5}}{20} & \frac{T^{4}}{8} & \frac{T^{3}}{6} \\
\frac{T^{5}}{30} & \frac{T^{4}}{8} & \frac{T^{3}}{3} & \frac{T^{2}}{2} \\
\frac{T^{4}}{24} & \frac{T^{3}}{6} & \frac{T^{2}}{2} & T
\end{array}\right]
$$

For example the element $Q_{44}$ in last row and lost column can be derived using L-Hospital rule as:

$$
Q_{44}==_{\alpha \rightarrow 0}^{L t} q_{44}={ }_{\alpha \rightarrow 0}^{L t} \frac{1}{2 \alpha}\left(1-e^{-2 \alpha T}\right)==_{\alpha \rightarrow 0}^{L t} \frac{\frac{d}{d \alpha}\left(1-e^{-2 \alpha T}\right)}{\frac{d}{d \alpha}(2 \alpha)}={ }_{\alpha \rightarrow 0}^{L t} \frac{2 T e^{-2 \alpha T}}{2}=T
$$

Similarly the rest of the elements can be derived.

\section{A. Extended Kalman Filter}

Generally, the EKF is done in two steps viz., time updation and measurement updation. In this report the time updation is done in Cartesian coordinates and measurement updation is done in spherical coordinates ${ }^{6}$.

Time updation:

Predicted state vector: $\tilde{X}(k \mid k-1)=\Phi \hat{X}(k-1 \mid k-1)$

Predicted state error covariance: $\tilde{P}(k \mid k-1)=\Phi \hat{P}(k-1 \mid k-1) \Phi^{T}+Q$

\section{Measurement updation:}

Measurement fusion is done by concatenation of measurement vector from imaging sensor \& radar and the corresponding observation matrices are appended to each other.

Predicted measurement: $\tilde{z}=\left[\begin{array}{lllll}\tilde{\theta} & \tilde{\varphi} & \tilde{\theta} & \tilde{\varphi} & \tilde{r}\end{array}\right]^{T}$ 


$$
\begin{aligned}
& \tilde{\theta}=\tan ^{-1}\left(\frac{\tilde{X}(1)}{\tilde{X}(5)}\right) \\
& \tilde{\varphi}=\tan ^{-1}\left(\frac{\tilde{X}(9)}{\sqrt{\tilde{X}(1)^{2}+\tilde{X}(5)^{2}}}\right) \\
& \tilde{r}=\sqrt{\tilde{X}(1)^{2}+\tilde{X}(5)^{2}+\tilde{X}(9)^{2}}
\end{aligned}
$$

where $\tilde{X}(1), \tilde{X}(5)$ and $\tilde{X}(9)$ are predicted $\mathrm{x}$-, y- and z-position respectively

$$
\text { Innovation: } e=z-\widetilde{z} \text {, where } Z=\left[\begin{array}{lllll}
\theta_{m}^{I} & \varphi_{m}^{I} & \theta_{m}^{R} & \varphi_{m}^{R} & r_{m}^{R}
\end{array}\right]
$$

The linearised system observation matrix $H$ is obtained:

where

$$
H=\left.\frac{\partial h}{\partial X}\right|_{X=\tilde{X}}=\left[\begin{array}{cccccccccccc}
\theta_{x} & 0 & 0 & 0 & \theta_{y} & 0 & 0 & 0 & 0 & 0 & 0 & 0 \\
\varphi_{x} & 0 & 0 & 0 & \varphi_{y} & 0 & 0 & 0 & \varphi_{z} & 0 & 0 & 0 \\
\theta_{x} & 0 & 0 & 0 & \theta_{y} & 0 & 0 & 0 & 0 & 0 & 0 & 0 \\
\varphi_{x} & 0 & 0 & 0 & \varphi_{y} & 0 & 0 & 0 & \varphi_{z} & 0 & 0 & 0 \\
r_{x} & 0 & 0 & 0 & r_{y} & 0 & 0 & 0 & r_{z} & 0 & 0 & 0
\end{array}\right]
$$

$$
\begin{array}{lll}
\theta_{x}=\frac{\cos (\tilde{\theta})}{\tilde{r} \cos (\tilde{\varphi})} & \theta_{y}=\frac{-\sin (\tilde{\theta})}{\tilde{r} \cos (\tilde{\varphi})} & \\
\varphi_{x}=\frac{-\sin (\tilde{\theta}) \sin (\tilde{\varphi})}{\tilde{r}} & \varphi_{y}=\frac{-\cos (\tilde{\theta}) \sin (\tilde{\varphi})}{\tilde{r}} & \varphi_{z}=\frac{\cos (\tilde{\varphi})}{\tilde{r}} \\
r_{x}=\sin (\tilde{\theta}) \cos (\tilde{\varphi}) & r_{y}=\cos (\tilde{\theta}) \cos (\tilde{\varphi}) & r_{z}=\sin (\tilde{\varphi})
\end{array}
$$

Innovation covariance matrix: $S=H \tilde{P}(k \mid k-1) H^{T}+R$

Kalman gain is computed as: $K=\tilde{P}(k \mid k-1) H^{T} S^{-1}$

Estimated state: $\hat{X}(k \mid k)=\tilde{X}(k \mid k-1)+K e$

State error covariance matrix: $\hat{P}(k \mid k)=(I-K H) \tilde{P}(k \mid k-1)$

\section{B. Extended Kalman Filter Initialization}

Ten percentage deviation from the true state vector could be used to initialize the position, velocity and acceleration states in EKF. The jerk state is initialized to zero. The initial state estimates are given by:

$$
\hat{X}=0.9 X_{t}
$$

where $\hat{X}$ : initial estimated state vector at scan number one

$\hat{X}_{t}$ : true state vector at scan number one

The expression for the initial state error covariance matrix is given by:

$$
\hat{P}=\operatorname{diag}\left[\left(X_{t}-\hat{X}\right)^{2}\right]
$$

Each of the elements in $P$ represents the uncertainty in the initial estimates of the states.

\section{Tracking in 3D Space}

The measurements are assumed to be available in spherical coordinates in terms of range, azimuth and elevation $(r, \theta, \varphi)$. The measurement and associated noise covariance are fused at very scan. The measurements come from IRST and radar, at each scan, are appended as a single vector as: 


$$
Z=\left[\begin{array}{lllll}
\theta_{m}^{I} & \varphi_{m}^{I} & \theta_{m}^{R} & \varphi_{m}^{R} & r_{m}^{R}
\end{array}\right]^{T}
$$

The associated noise variances are concatenated as a single matrix as:

$$
R=\left[\begin{array}{ccccc}
\left(\sigma_{\theta}^{I}\right)^{2} & 0 & 0 & 0 & 0 \\
0 & \left(\sigma_{\varphi}^{I}\right)^{2} & 0 & 0 & 0 \\
0 & 0 & \left(\sigma_{\theta}^{R}\right)^{2} & 0 & 0 \\
0 & 0 & 0 & \left(\sigma_{\varphi}^{R}\right)^{2} & 0 \\
0 & 0 & 0 & 0 & \left(\sigma_{r}^{R}\right)^{2}
\end{array}\right]
$$

\section{A. Acceleration Model}

The general form of the acceleration model is given by:

$$
\begin{aligned}
& X_{a}(k+1)=\Phi_{a} X_{a}(k)+w_{a}(k) \\
& Z_{a}(k+1)=h\left(X_{a}(k)\right)+v_{a}(k+1)
\end{aligned}
$$

where $X_{a}=\left[\begin{array}{lllllllll}x & \dot{x} & \ddot{x} & y & \dot{y} & \ddot{y} & z & \dot{z} & \ddot{z}\end{array}\right]^{T}$

$w_{a}=\left[\begin{array}{lllllllll}u_{1 a} & u_{2 a} & u_{3 a} & u_{4 a} & u_{5 a} & u_{6 a} & u_{7 a} & u_{8 a} & u_{9 a}\end{array}\right]^{T}$

$u_{1 a}, u_{2 a}, \ldots$ are the driving noise components for the acceleration model.

The state transition and noise covariance matrices for the acceleration model are:

$$
\begin{array}{rlrl}
\Phi_{a} & =\left[\begin{array}{ccc}
\phi_{a} & 0 & 0 \\
0 & \phi_{a} & 0 \\
0 & 0 & \phi_{a}
\end{array}\right] & Q_{a}=2 \alpha \sigma_{m}^{2}\left[\begin{array}{ccc}
\varsigma_{a} & 0 & 0 \\
0 & \varsigma_{a} & 0 \\
0 & 0 & \varsigma_{a}
\end{array}\right] \\
\text { where } \phi_{a}=\left[\begin{array}{lll}
1 & T & q \\
0 & 1 & r \\
0 & 0 & s
\end{array}\right] & \varsigma_{a}=\left[\begin{array}{lll}
q_{22} & q_{23} & q_{24} \\
q_{32} & q_{33} & q_{34} \\
q_{42} & q_{43} & q_{44}
\end{array}\right]
\end{array}
$$

\section{B. Jerk Model}

The jerk model has the general form

$$
\begin{gathered}
X_{j}(k+1)=\Phi_{j} X_{j}(k)+w_{j}(k) \\
Z_{j}(k+1)=h\left(X_{j}(k)\right)+v_{j}(k+1) \\
\text { where } X_{j}=\left[\begin{array}{llllllllllll}
x & \dot{x} & \ddot{x} & \dddot{x} & y & \dot{y} & \ddot{y} & \dddot{y} & z & \dot{z} & \ddot{z} & \dddot{z}
\end{array}\right]^{T}
\end{gathered}
$$$$
w_{j}=\left[\begin{array}{llllllllllll}
u_{1 j} & u_{2 j} & u_{3 j} & u_{4 j} & u_{5 j} & u_{6 j} & u_{7 j} & u_{8 j} & u_{9 j} & u_{10 j} & u_{11 j} & u_{12 j}
\end{array}\right]^{T}
$$$$
u_{1 j}, u_{2 j}, \ldots \text { are the driving noise components for jerk model. }
$$

The state transition and noise covariance matrices for the jerk model are:

$$
\Phi_{j}=\left[\begin{array}{ccc}
\phi_{j} & 0 & 0 \\
0 & \phi_{j} & 0 \\
0 & 0 & \phi_{j}
\end{array}\right] \quad Q_{j}=2 \alpha \sigma_{j}^{2}\left[\begin{array}{ccc}
\varsigma_{j} & 0 & 0 \\
0 & \varsigma_{j} & 0 \\
0 & 0 & \varsigma_{j}
\end{array}\right]
$$




$$
\text { where } \phi_{j}=\left[\begin{array}{cccc}
1 & T & \frac{T^{2}}{2} & p \\
0 & 1 & T & q \\
0 & 0 & 1 & r \\
0 & 0 & 0 & s
\end{array}\right] \varsigma_{j}=\left[\begin{array}{llll}
q_{11} & q_{12} & q_{13} & q_{14} \\
q_{21} & q_{22} & q_{23} & q_{24} \\
q_{31} & q_{32} & q_{33} & q_{34} \\
q_{41} & q_{42} & q_{43} & q_{44}
\end{array}\right]
$$

\section{Results and Discussion}

The tracking performance of the EKF with an acceleration model (eqs.38-39) and jerk model in the states (eqs.40-41) is illustrated with simulated data of a target subjected to constant jerk ${ }^{1}$.

Performance of four-state jerk model with three state acceleration model is compared with simulated data. The sampling time $T$ is $0.5 \mathrm{sec}$. The target starts at position $(0,0,100)$ with constant velocity of $-100 \mathrm{~m} / \mathrm{s}$ in $\mathrm{x}$-axis and $10 \mathrm{~m} / \mathrm{s}$ in $\mathrm{y}$-axis. A step jerk of $0.009 \mathrm{~m} / \mathrm{s}^{3}$ is applied along $\mathrm{x}$-axis at $50 \mathrm{~s}$ and $-0.009 \mathrm{~m} / \mathrm{s}^{3}$ is applied along $\mathrm{z}$-axis at 125 s, which results in a ramp acceleration, parabola velocity and cubic position variation.

Random noises with the following variances are added to range, azimuth and elevation to generate noisy measurement data:

Measurement noise variances

$$
\begin{aligned}
\text { for IRST: } & \left(\sigma_{\theta}^{I}\right)^{2} 5 \mathrm{e}-5 \mathrm{rad}^{2} \text { in azimuth } \\
& \left(\sigma_{\varphi}^{I}\right)^{2} \text { 25e-5 } \mathrm{rad}^{2} \text { in elevation } \\
\text { for radar: } & \left(\sigma_{r}^{R}\right)^{2} 2.25 \mathrm{e} 3 \mathrm{~m}^{2} \text { in range } \\
& \left(\sigma_{\theta}^{R}\right)^{2} 5 \mathrm{e}-3 \mathrm{rad}^{2} \text { in azimuth } \\
& \left(\sigma_{\varphi}^{R}\right)^{2} 25 \mathrm{e}-3 \mathrm{rad}^{2} \text { in elevation }
\end{aligned}
$$

Correlation factor is chosen as $\alpha=0.006$ for both the models. Although in the acceleration model it is the target acceleration that is correlated and in the jerk model it is the target jerk that is correlated, the same $\alpha$ is used to describe both the models to maintain a degree of analytical similarity between the two models.

Process noise variance of $Q_{a}=2 \alpha \sigma_{m}^{2}, \sigma_{m}=1.8 \mathrm{~m} / \mathrm{s}^{2}$ is used for acceleration model. For the jerk model, process noise variance of $Q_{j}=2 \alpha \sigma_{j}^{2}, \sigma_{j}=0.009 \mathrm{~m} / \mathrm{s}^{3}$ is used. The simulated trajectory in x-axis is shown in Fig.1 and the measurements in spherical coordinates are shown in Fig.2.

The performances of both the models are evaluated with the average of seventy-five Monte Carlo simulations. Figs 3-5 show the estimated and true trajectories. It is observed that jerk model performed better. Fig.6 shows the root sum square error in position, velocity and acceleration. It is observed that the acceleration estimates are very good with jerk model and position estimates are almost similar. This shows the necessity of jerk model where ever good acceleration estimates is required. The percentage fit error and root mean square error in position, velocity and acceleration are shown in Table- 1 and mean absolute error in position, velocity and acceleration are shown in Table2. From these tables, it is observed that jerk model performed very well. The innovation sequences along with theoretical bounds are shown in Fig.7. Autocorrelation of residuals with bounds are shown in Fig.8 for whiteness test. It shows that both models satisfy the whiteness test. The state errors with bounds are shown in Figs. 9-11. It is observed that, the uncertainty in the state estimates is less in case of jerk model. Absolute errors in position, velocity and acceleration are shown in Figs. 12-14. It is observed that absolute errors are less with the jerk model of all sates with the improvement being most significant for the acceleration states. The norm and trace of the state error covariance matrix are shown in Fig. 15. It is observed that the norm and trace of the state error covariance matrix are less in case of jerk model that shows that the uncertainty in estimated states is less. The NEES and NIS are shown in Fig. 16. The average value of NIS is almost five and equal to the order of measurement vector that shows the robustness of the filter. It is learned that EKF filter would never satisfy the NEES criteria.

From the results it is clear that even for this case of simulated data, where the maneuver is slow and the acceleration is not very high, the acceleration errors are lesser when jerk model is used for tracking. It is seen that acceleration models fail to converge or result in large biases when the maneuvers are more vigorous whereas jerk models are seen to give good tracking performance under large maneuvers. 


\section{Concluding Remarks}

Jerk models, which include derivatives of position up to the third order, are used for tracking maneuvering targets. The performance of an extended Kalman filter with the jerk model is evaluated and compared with that of an EKF with acceleration model for simulated data of a target undergoing slow maneuvers. Detailed derivations and expressions for the jerk model in one/three dimensions and process noise covariances are presented. Measurement fusion strategy had been adopted to fuse the IRST and radar data. Simulation results clearly indicate that the tracking performance of the jerk model is superior to that of the acceleration model even when the maneuver is slow. It is expected that the performance improvement will be more significant when the target undergoes agile maneuvers.

\section{References}

${ }^{1}$ Kishore Mehrotra and Pravas R. Mahapatra, “A Jerk Model for Tracking Highly Maneuvering Targets”, IEEE Trans. on Aero. and Elec. Sys., AES-33, pp 1094-1105, Oct. 1997.

${ }^{2}$ VPS Naidu and Girija G., "Acceleration and jerk Models for Tracking Maneuvering targets”, NAL PD FC 0506, March 2006.

${ }^{3}$ Singer R.A., “Estimating Optimal Tracking Filter Performance for Manned Maneuvering Targets”, IEEE Trans. on Aero. and Elec. Sys., AES-7, pp 100-110, Jan. 1970.

${ }^{4}$ X. Ring LI and Vesselin P. Jilkov, "Survey of Maneuvering Target Tracking. Part I: Dynamic Models", IEEE Trans. on Aero. and Elec. Sys., AES-39, pp 1333-1364, Oct. 2003.

${ }^{5}$ Yaakov Bar-Shalom and Xiao-Rong Li, “Multitarget-Multisensor Tracking: Principles and Techniques”, 1995.

Table 1. Percentage fit error and root mean square errors in position, velocity and acceleration

\begin{tabular}{|c|c|c|c|c|c|c|}
\hline & \multicolumn{3}{|c|}{ PFE } & RMSPE & RMSVE & RMSAE \\
\cline { 2 - 4 } & $\mathrm{x}$ & $\mathrm{y}$ & $\mathrm{z}$ & & & \\
\hline jerk model & 0.13 & 1.06 & 4.42 & 4.15 & 0.55 & 0.03 \\
\hline acc. model & 0.16 & 1.74 & 6.38 & 3.13 & 0.55 & 0.06 \\
\hline
\end{tabular}

Table 2. Mean absolute errors in position, velocity and acceleration

\begin{tabular}{|l|l|l|l|l|l|l|l|l|l|}
\hline & \multicolumn{3}{|l|}{ in positions } & \multicolumn{3}{l|}{ in velocities } & \multicolumn{3}{l|}{ in accelerations } \\
\cline { 2 - 11 } & $\mathrm{x}$ & $\mathrm{y}$ & $\mathrm{z}$ & $\mathrm{x}$ & $\mathrm{y}$ & $\mathrm{z}$ & $\mathrm{x}$ & $\mathrm{y}$ & $\mathrm{z}$ \\
\hline jerk model & 11.31 & 14.31 & 29.08 & 1.2 & 1.14 & 2.12 & 0.07 & 0.05 & 0.1 \\
\hline acc. model & 14.3 & 19.39 & 37.65 & 1.2 & 1.14 & 4.18 & 0.22 & 0.2 & 0.25 \\
\hline
\end{tabular}
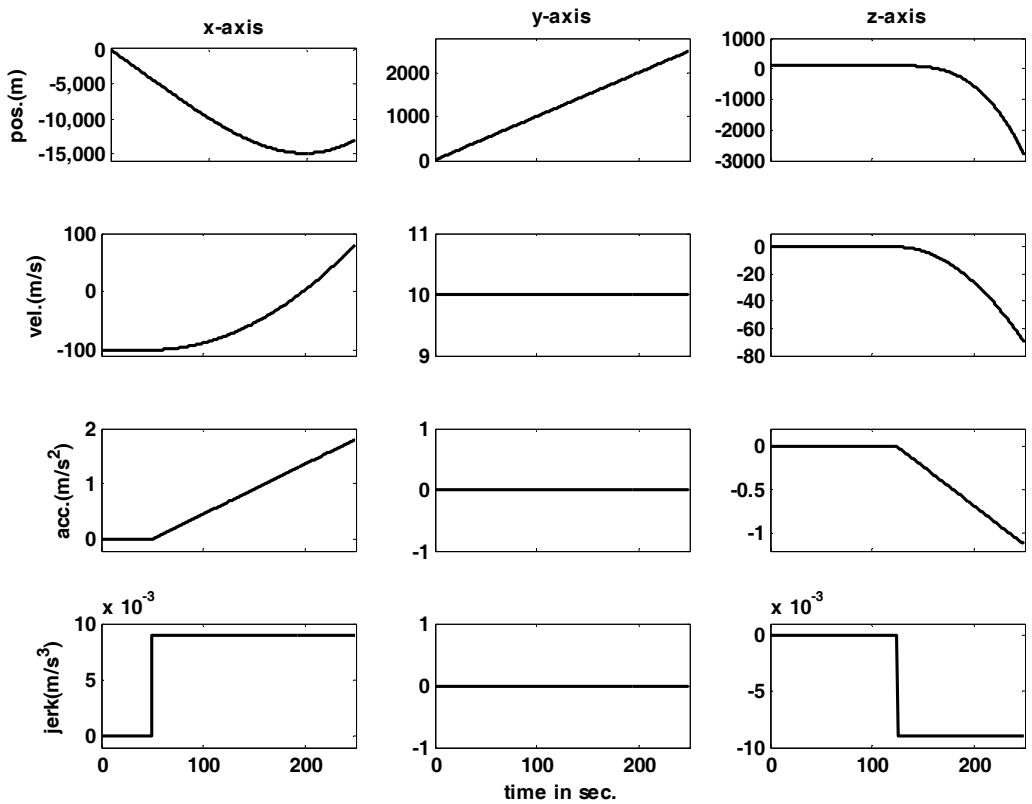

Figure 1. Simulated trajectory in $x-, y$ - and $z$-axis in Cartesian coordinated system 

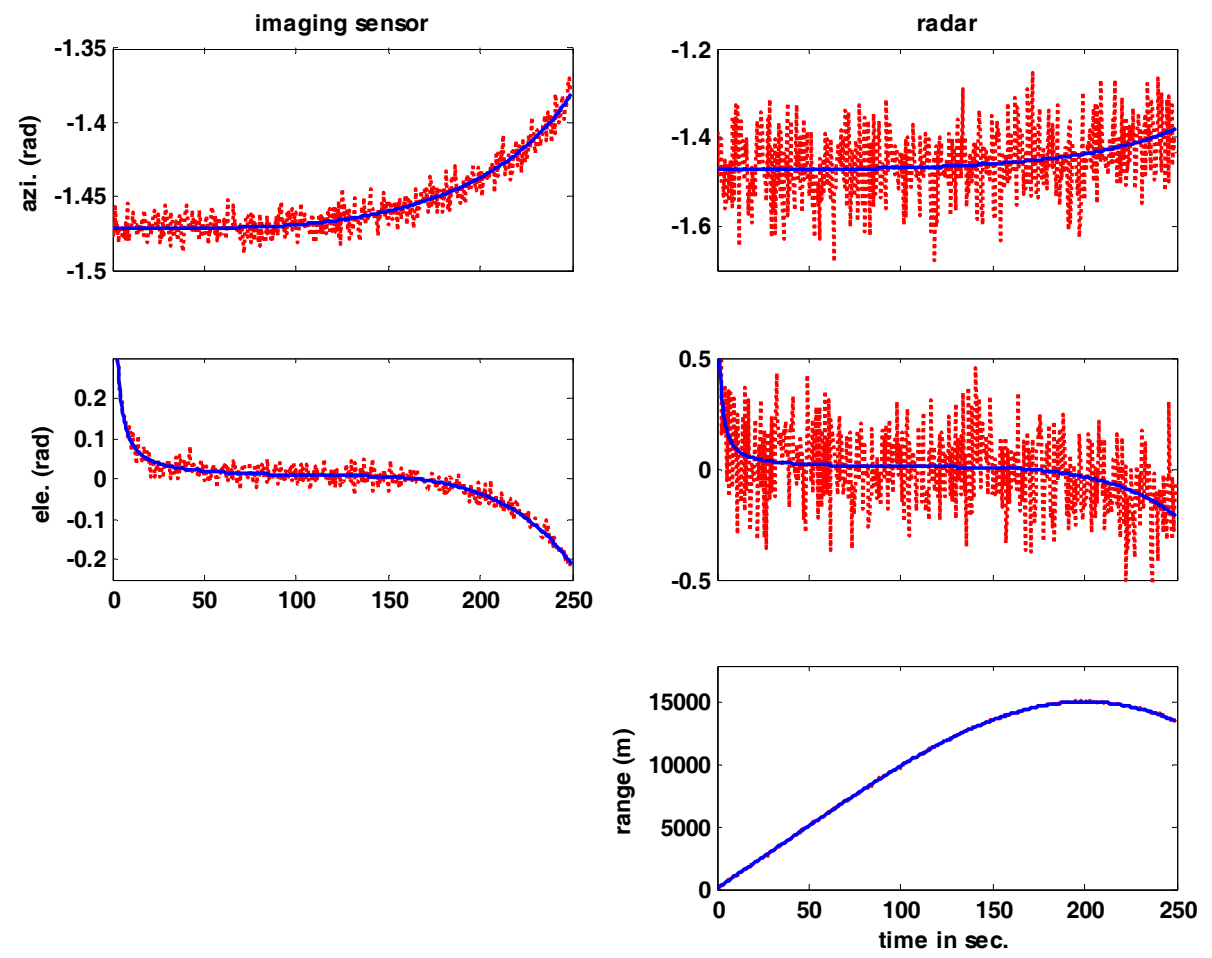

Figure 2. Measurements in spherical coordinates
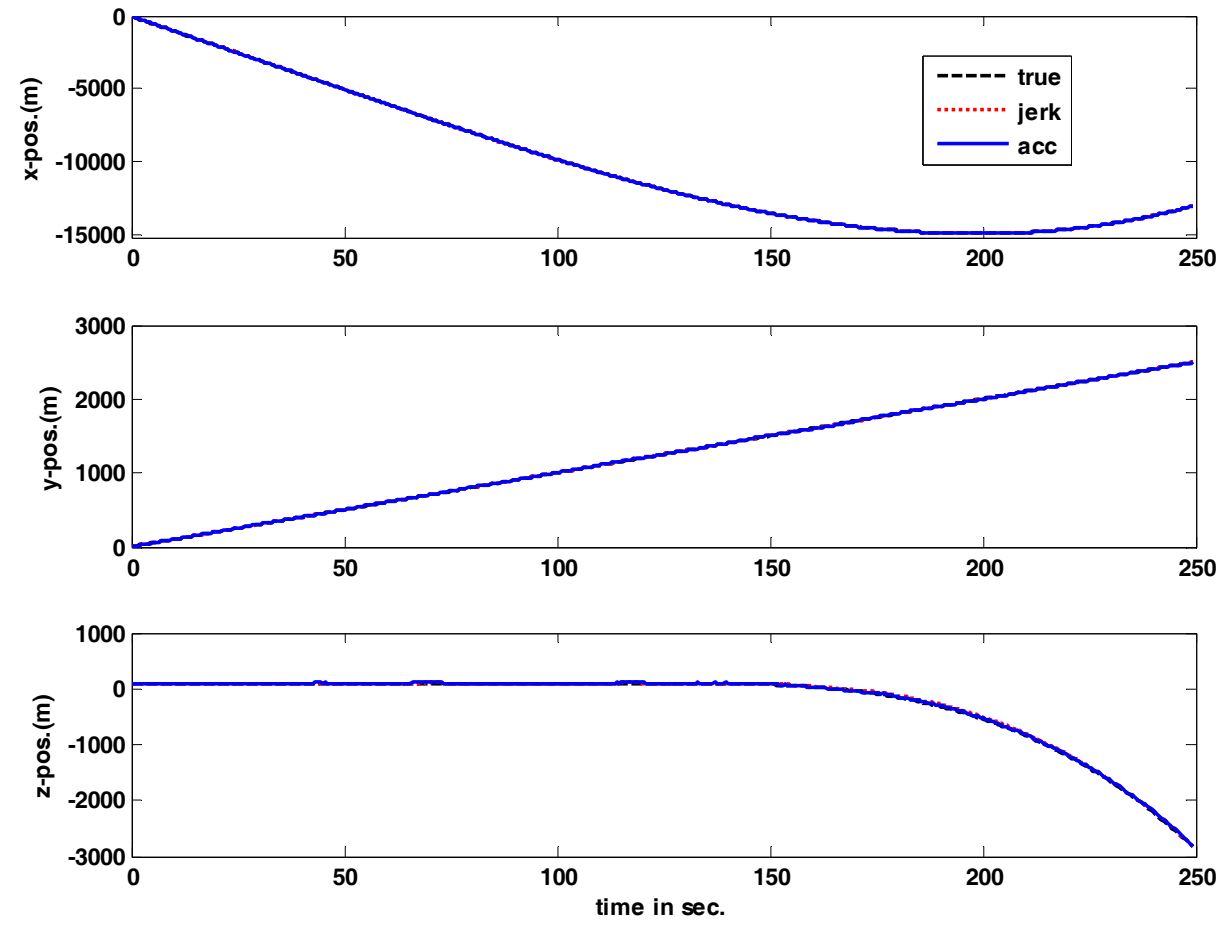

Figure 3. Estimated and true $x-, y$ - and z-position states 

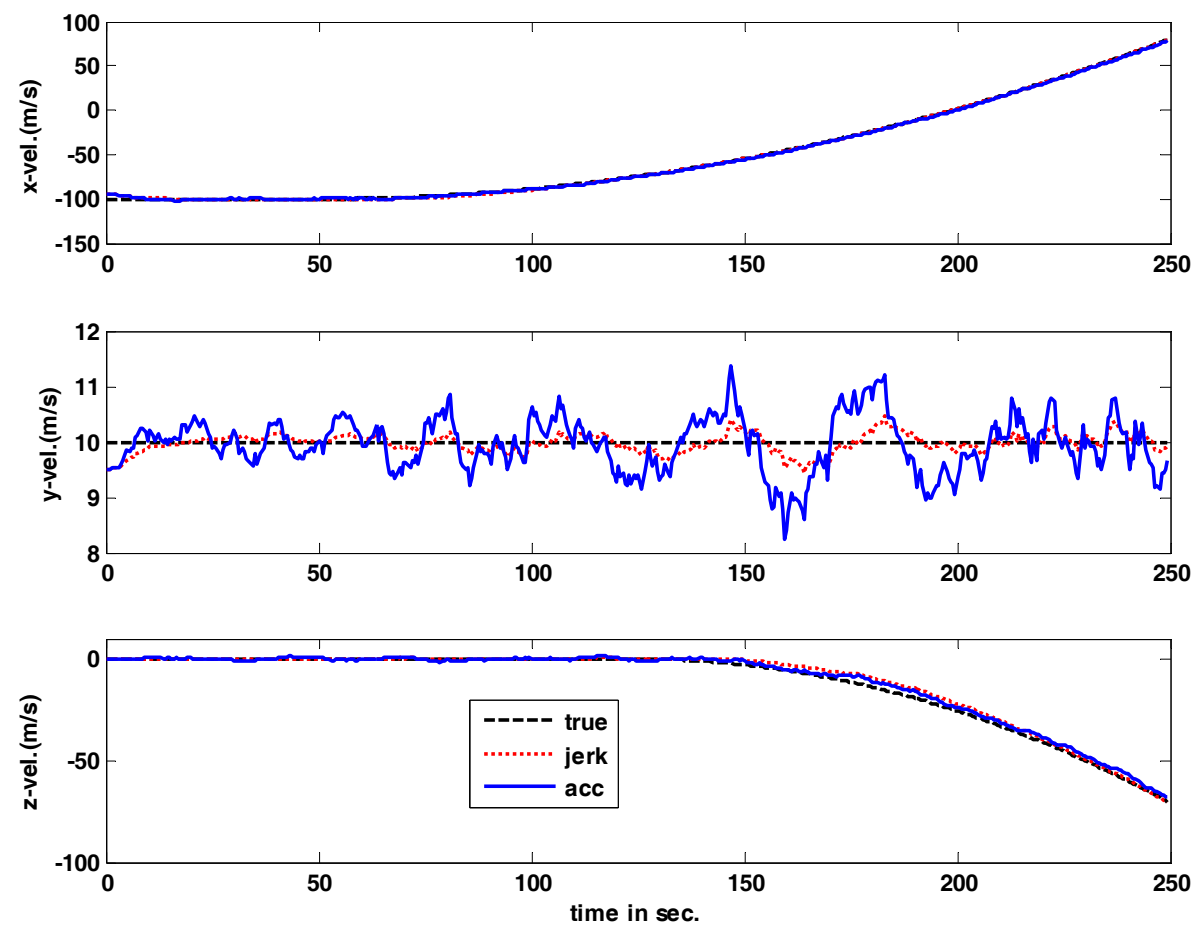

Figure 4. Estimated and true $x-, y$ - and z-velocity states
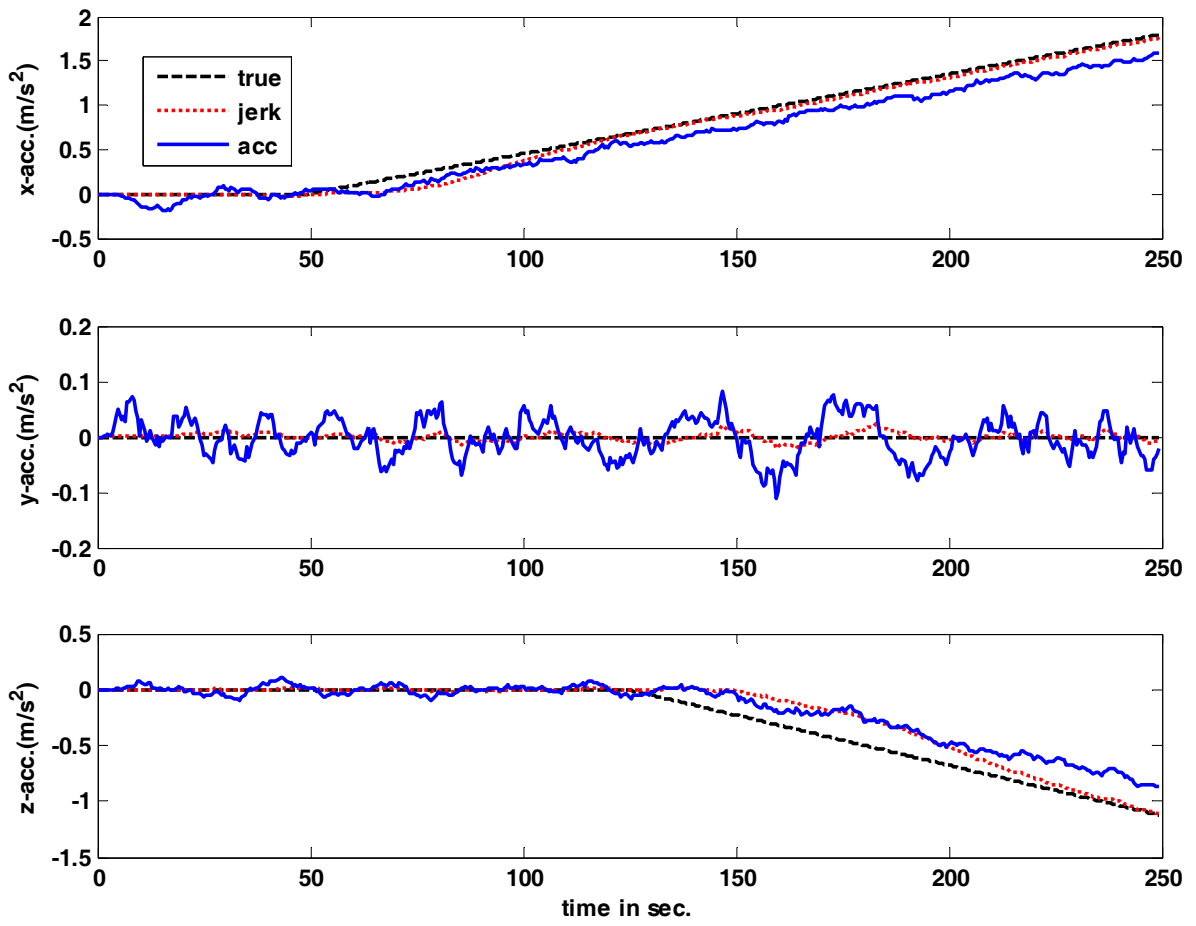

Figure 5. Estimated and true $x-, y$ - and z-acceleration states 

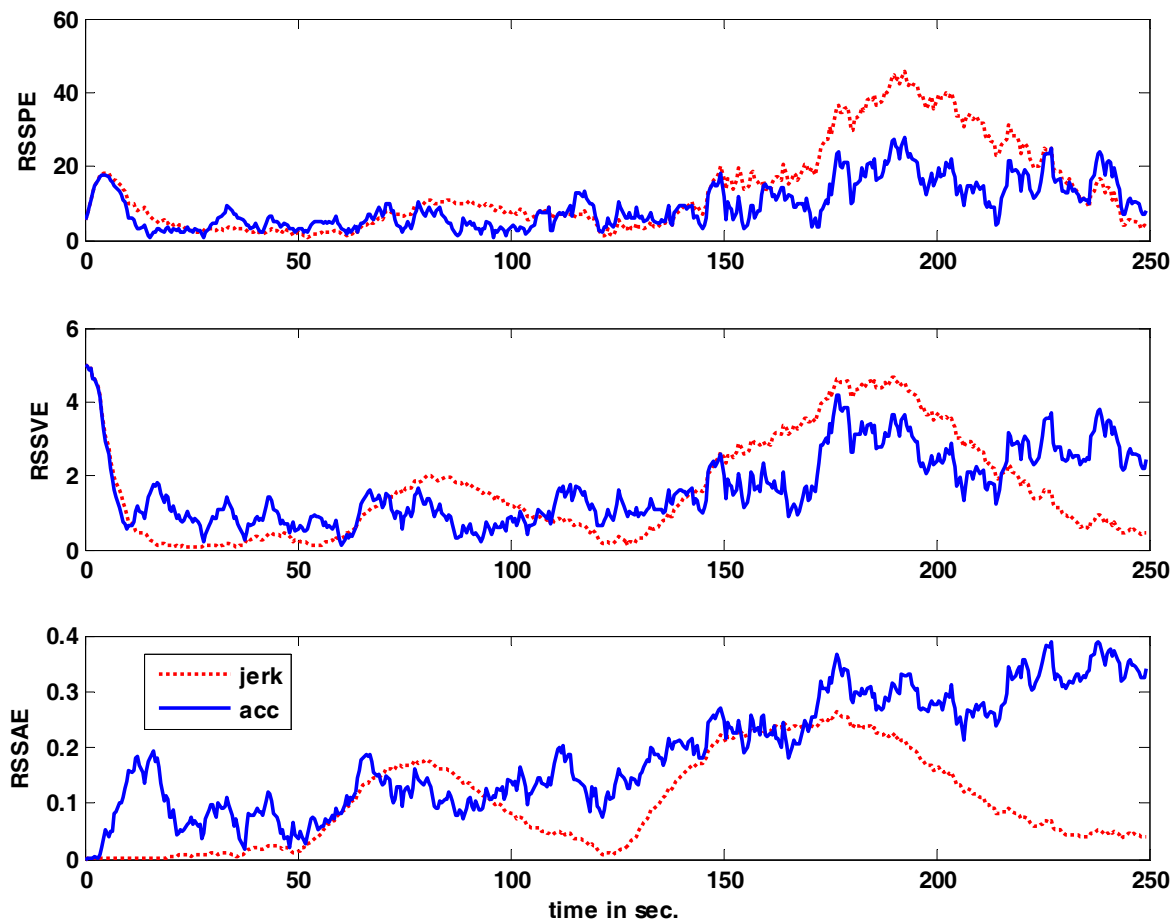

Figure 6. Root sum square errors in position, velocity and acceleration
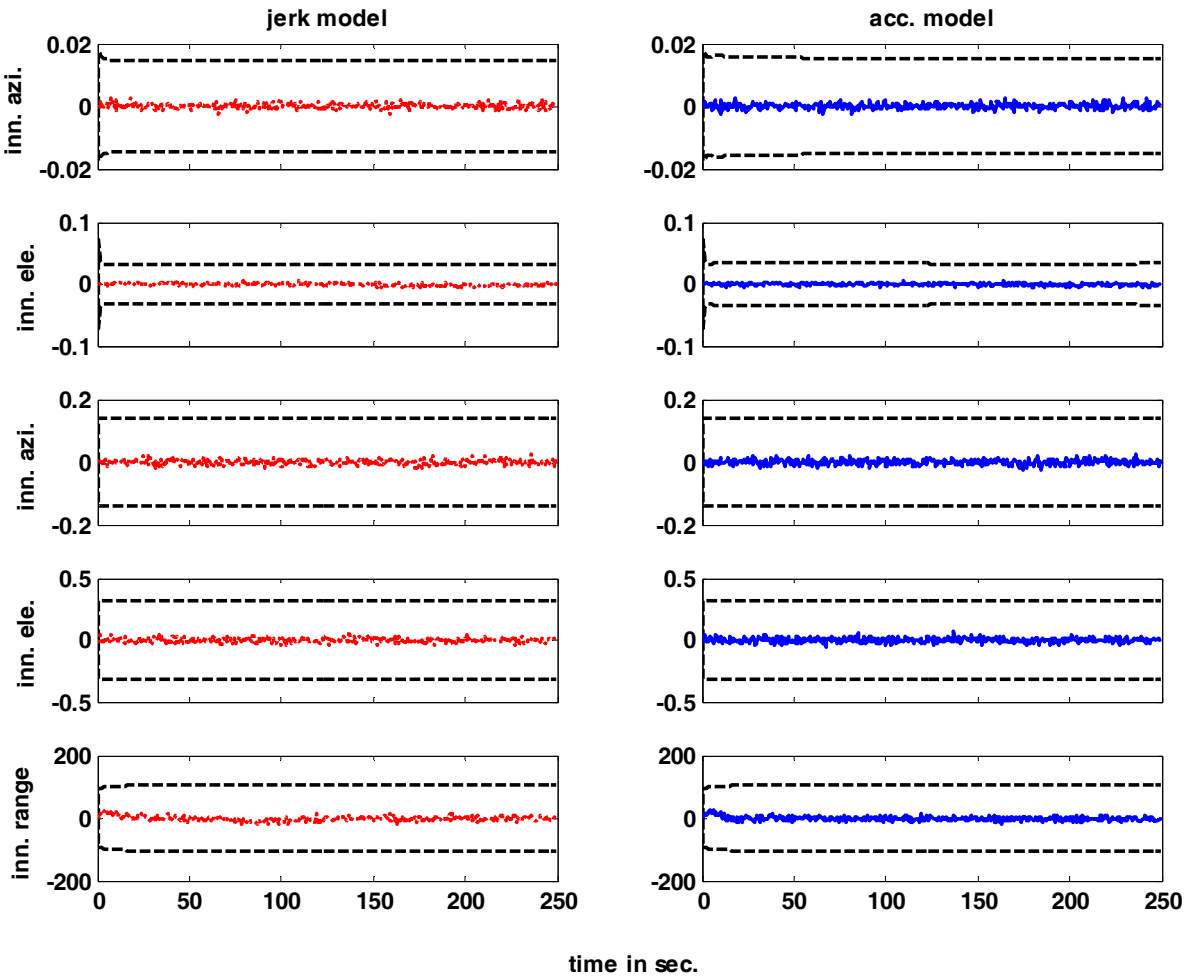

Figure 7. Innovation sequence with theoretical bounds 

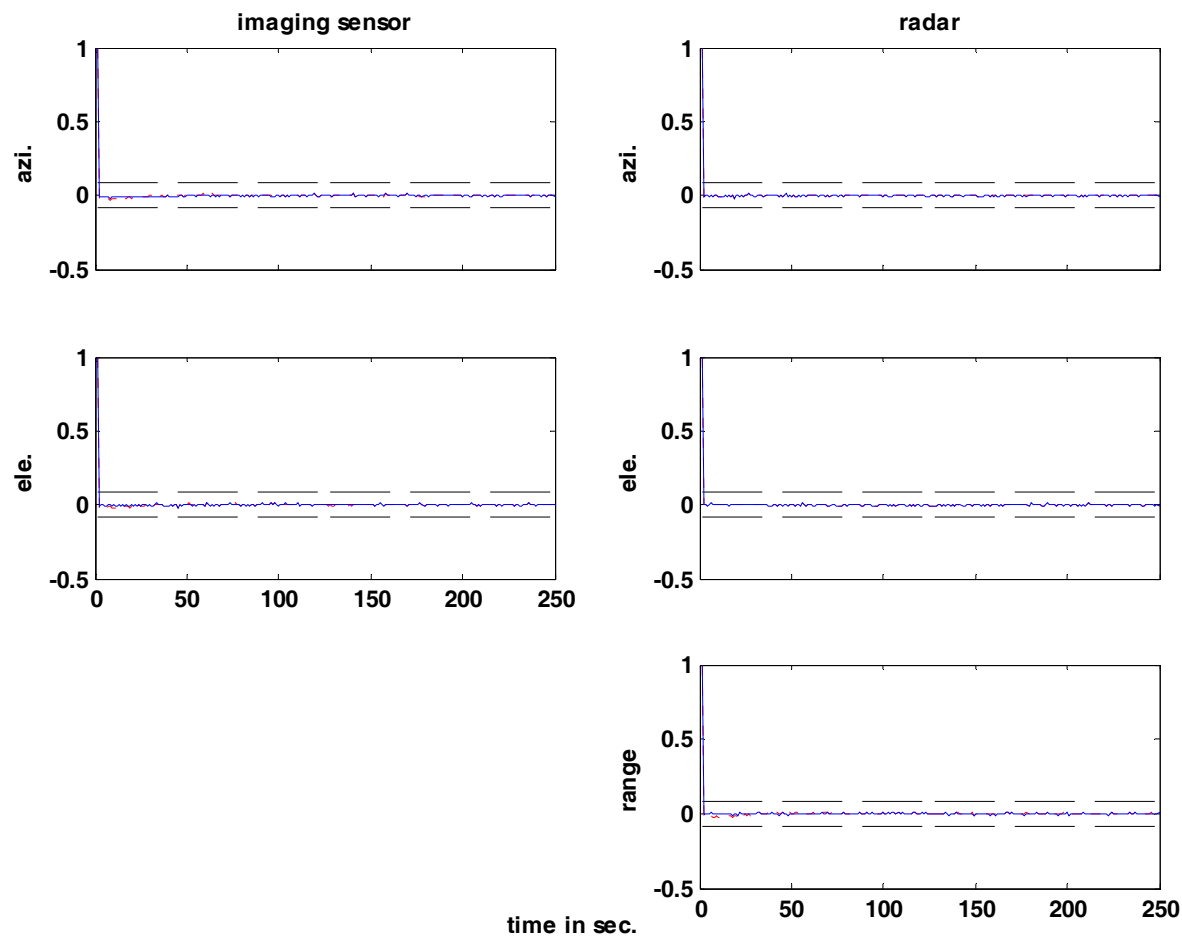

Figure 8. Autocorrelation of azimuth, elevation and range residuals with bounds
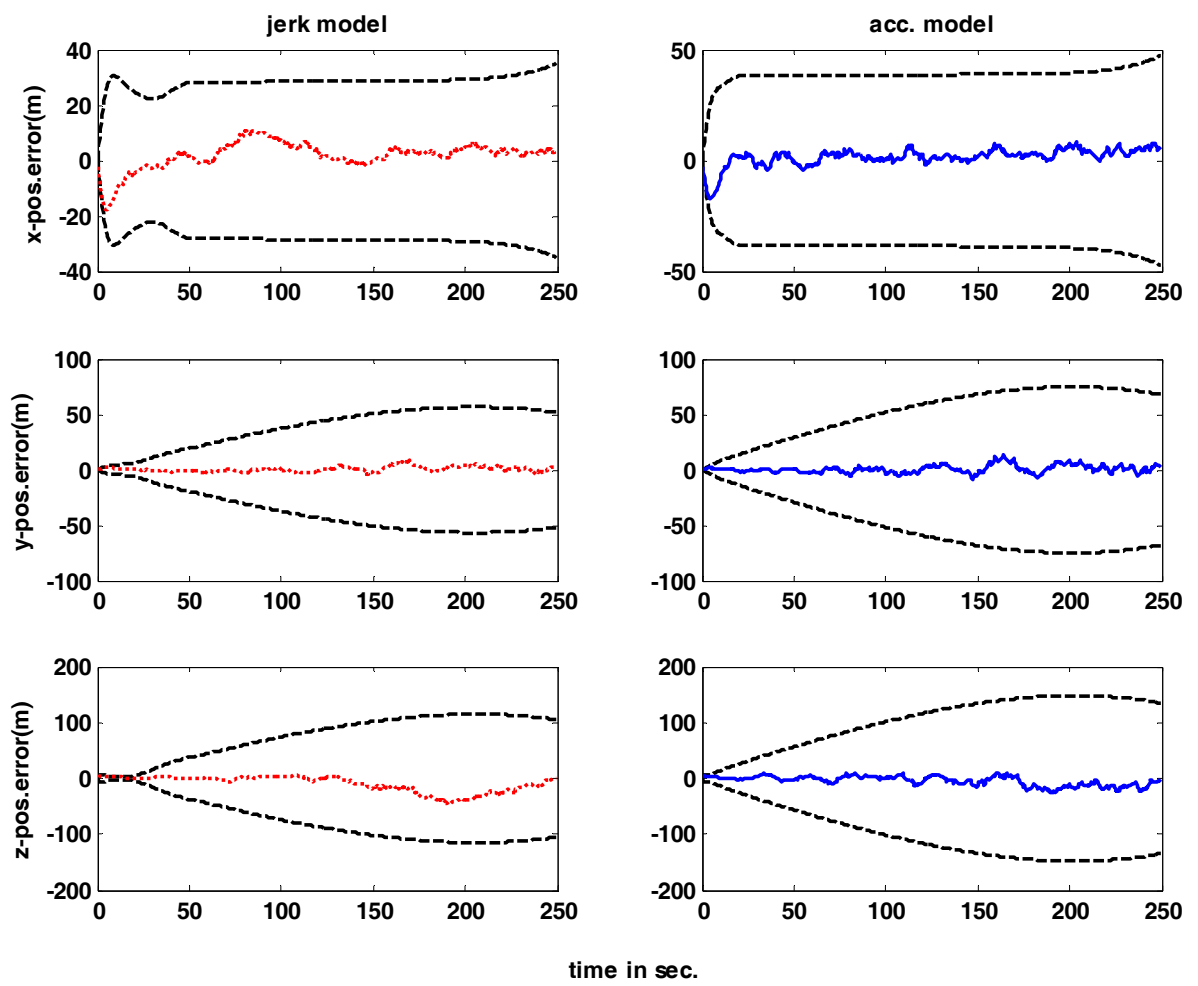

Figure 9. Position state errors with theoretical bounds 

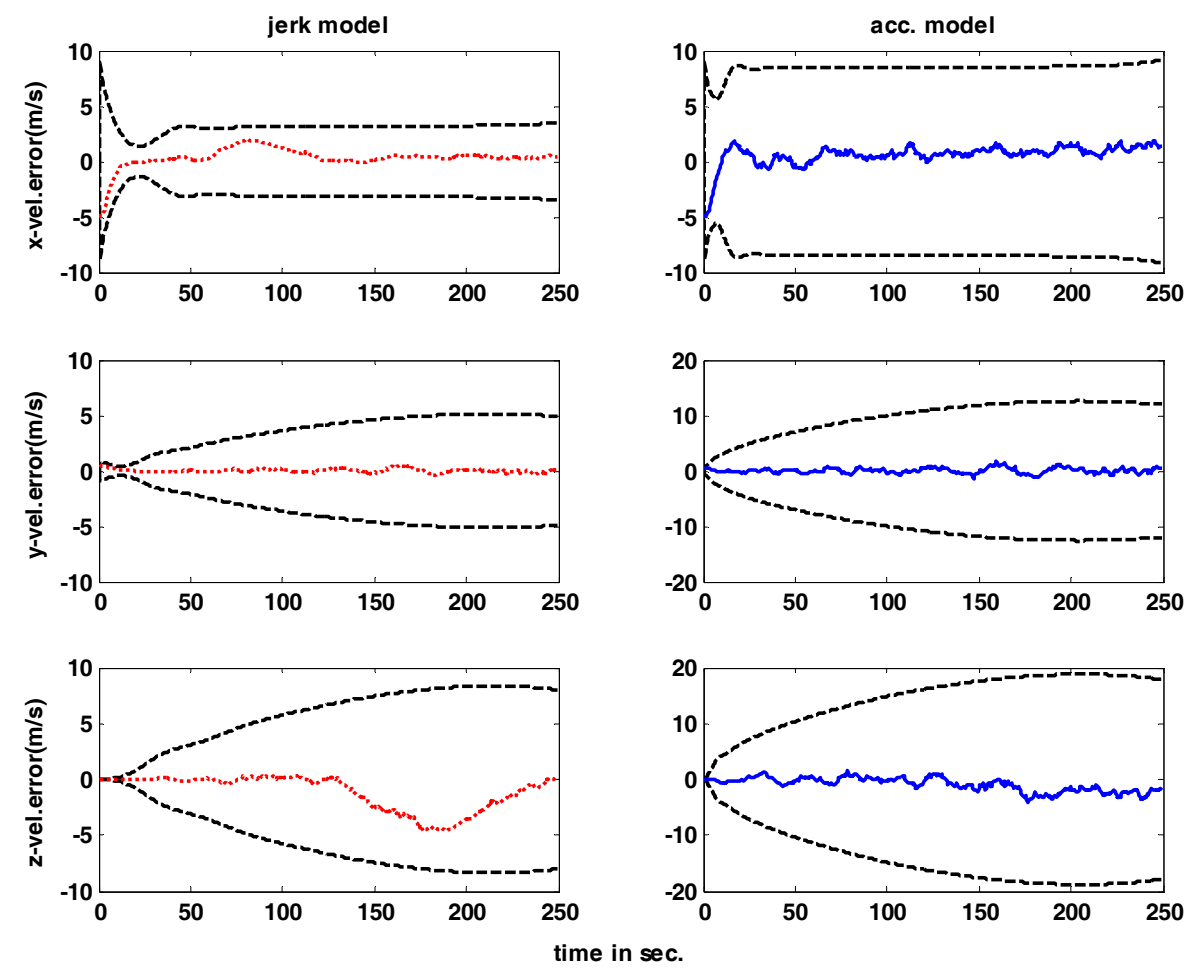

Figure 10. Velocity state errors with theoretical bounds
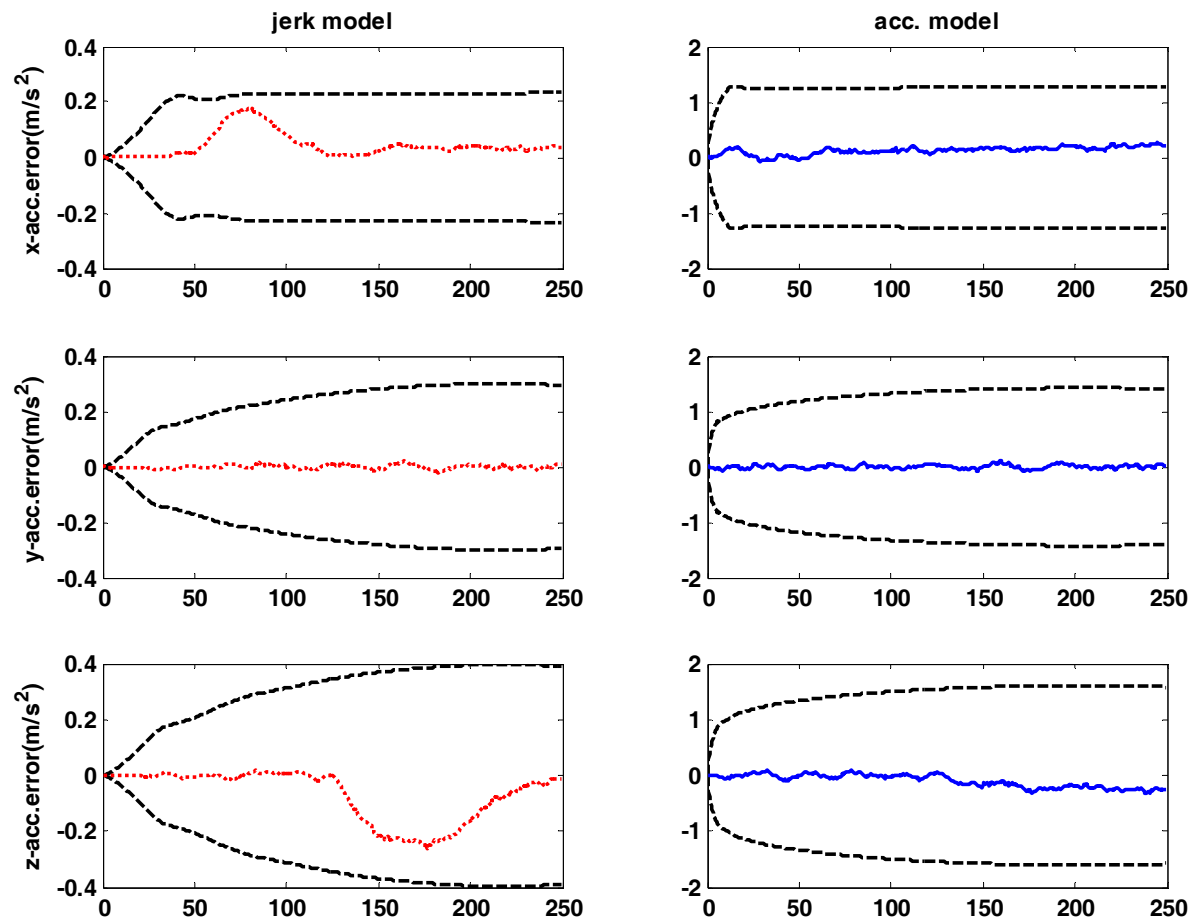

Figure 11. Acceleration state errors with theoretical bounds

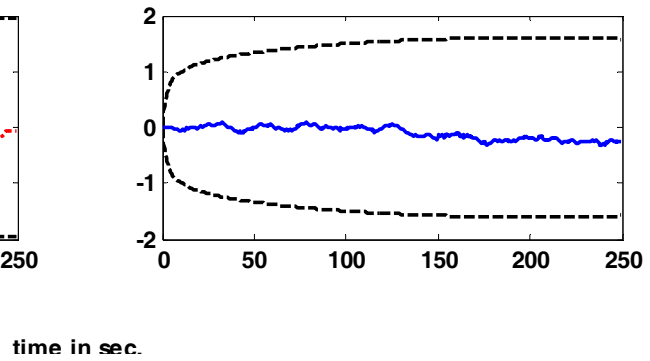



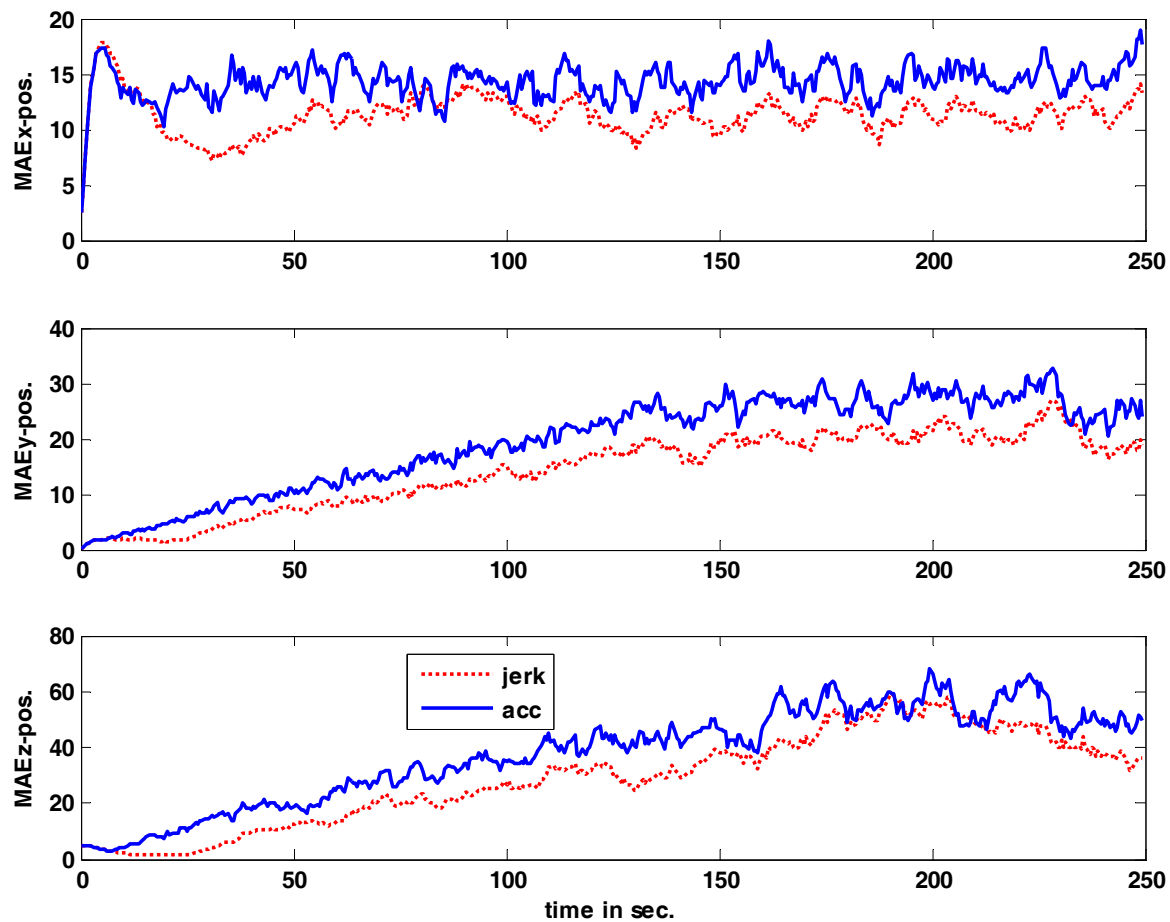

Figure 12. Absolute position error is $x-, y$ - and $z$-axis
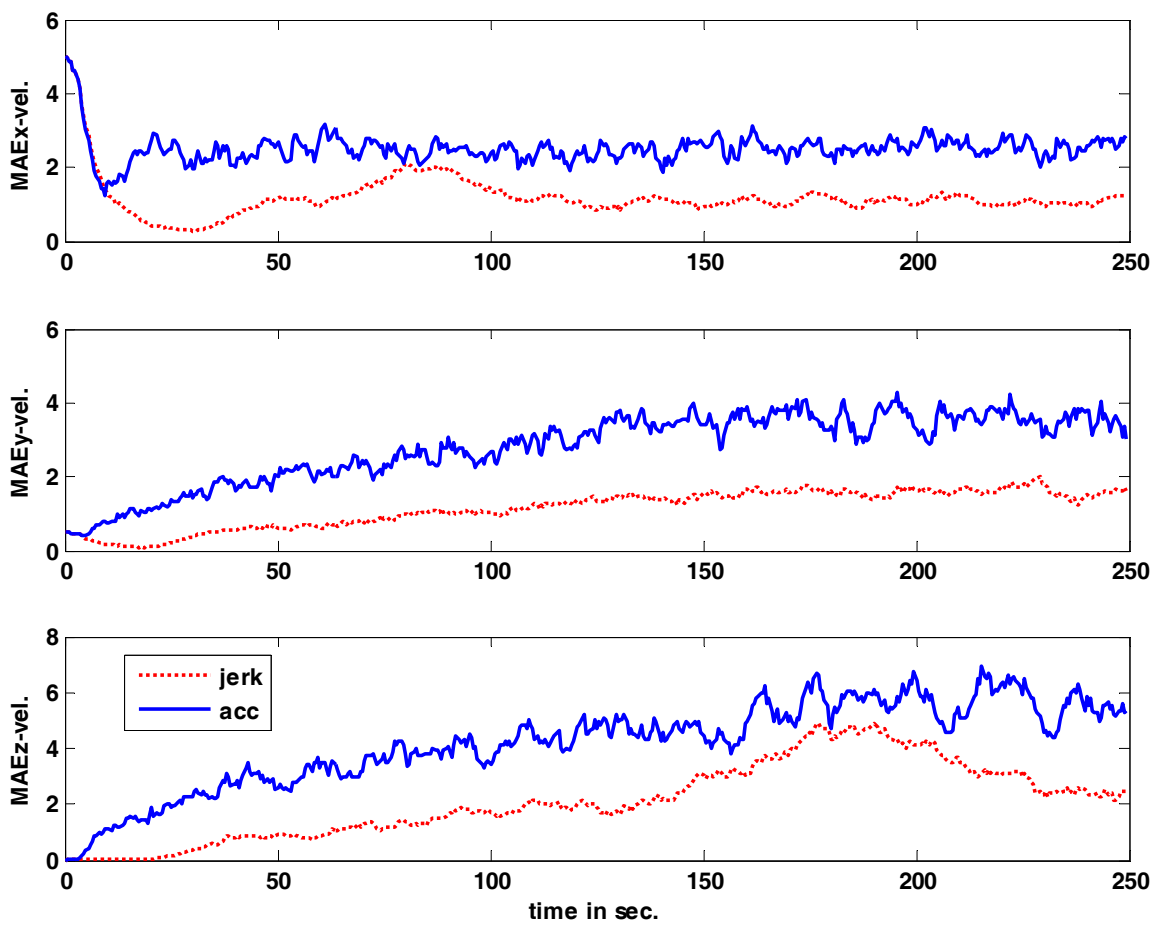

Figure 13. Absolute velocity error in $x-, y$ - and $z$-axis 

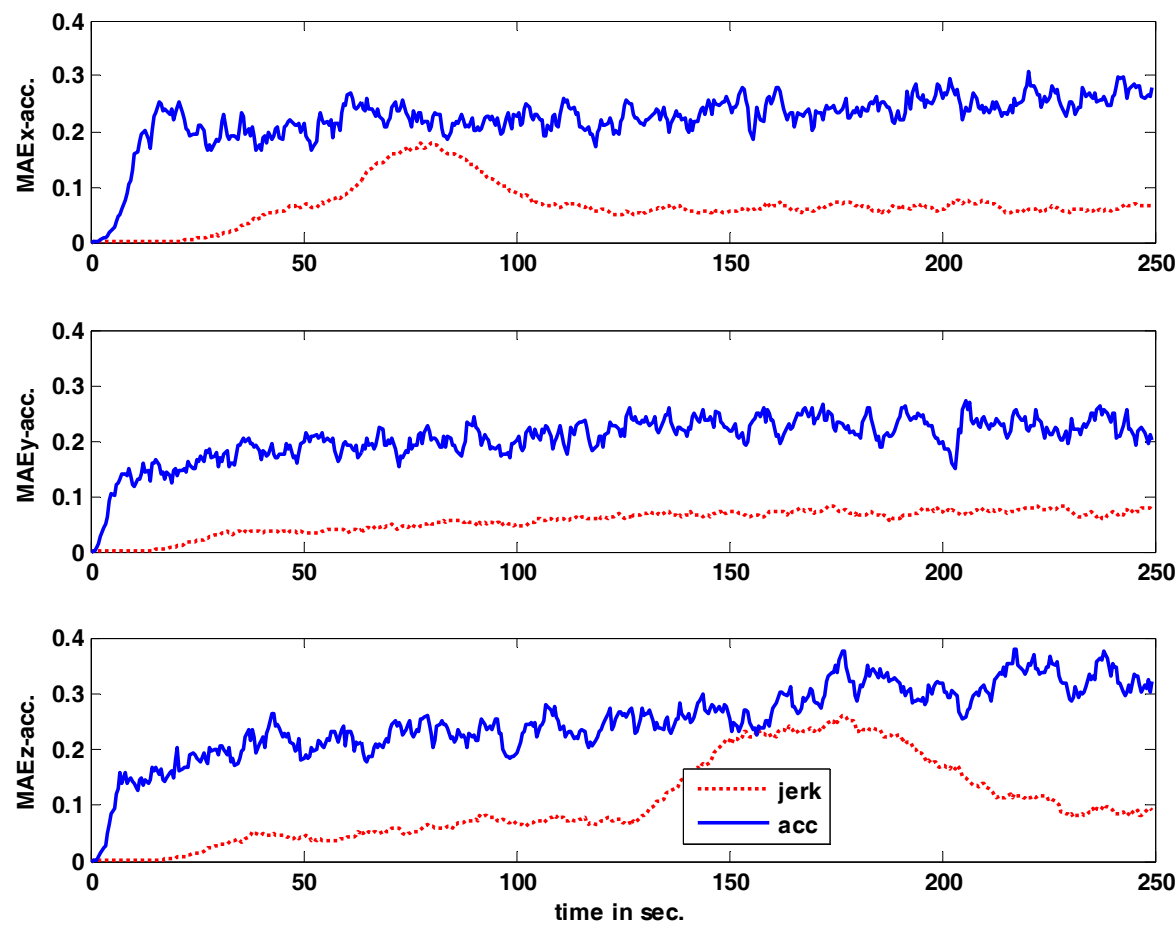

Figure 14. Absolute acceleration error in $x-, y$ - and $z$-axis
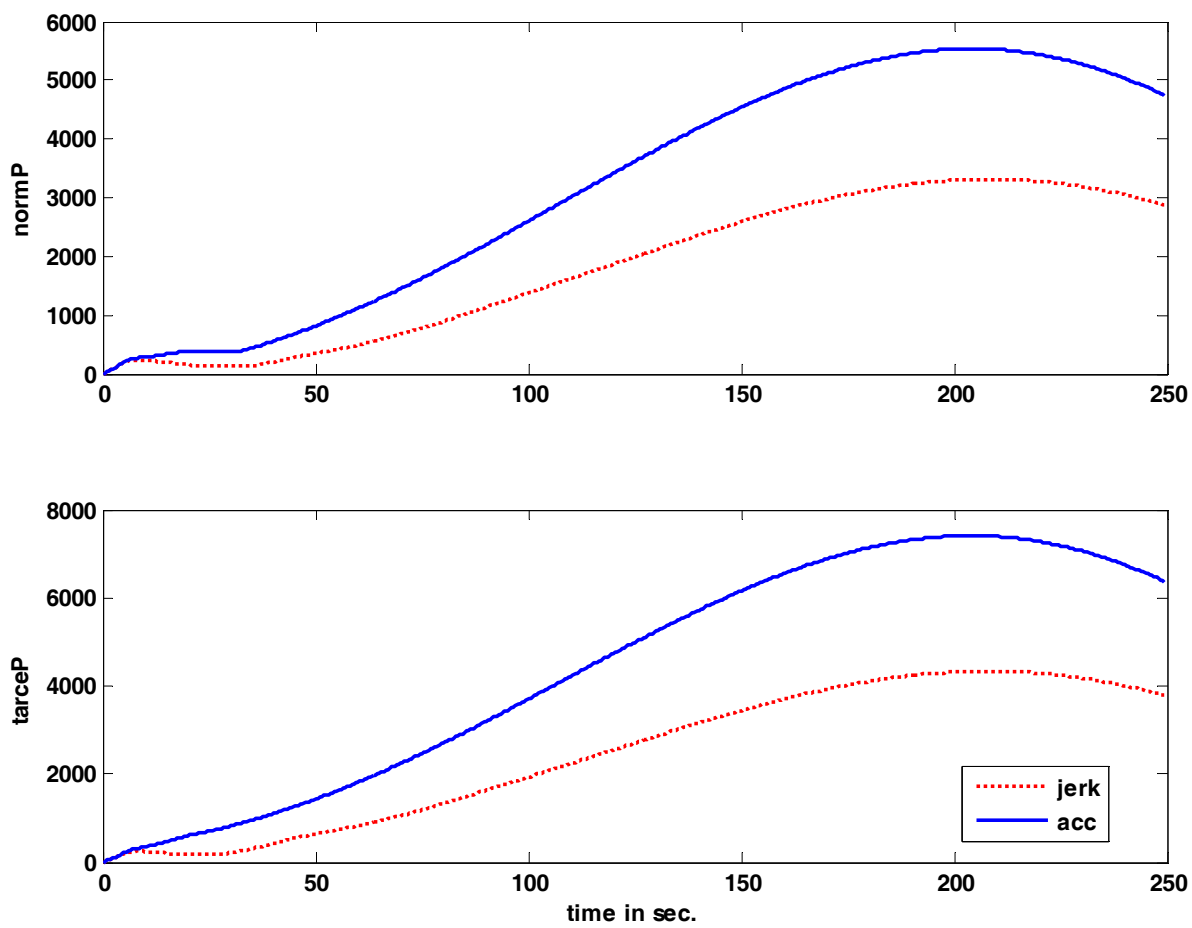

Figure 15. Norm and trace of the state error covariance matrix 

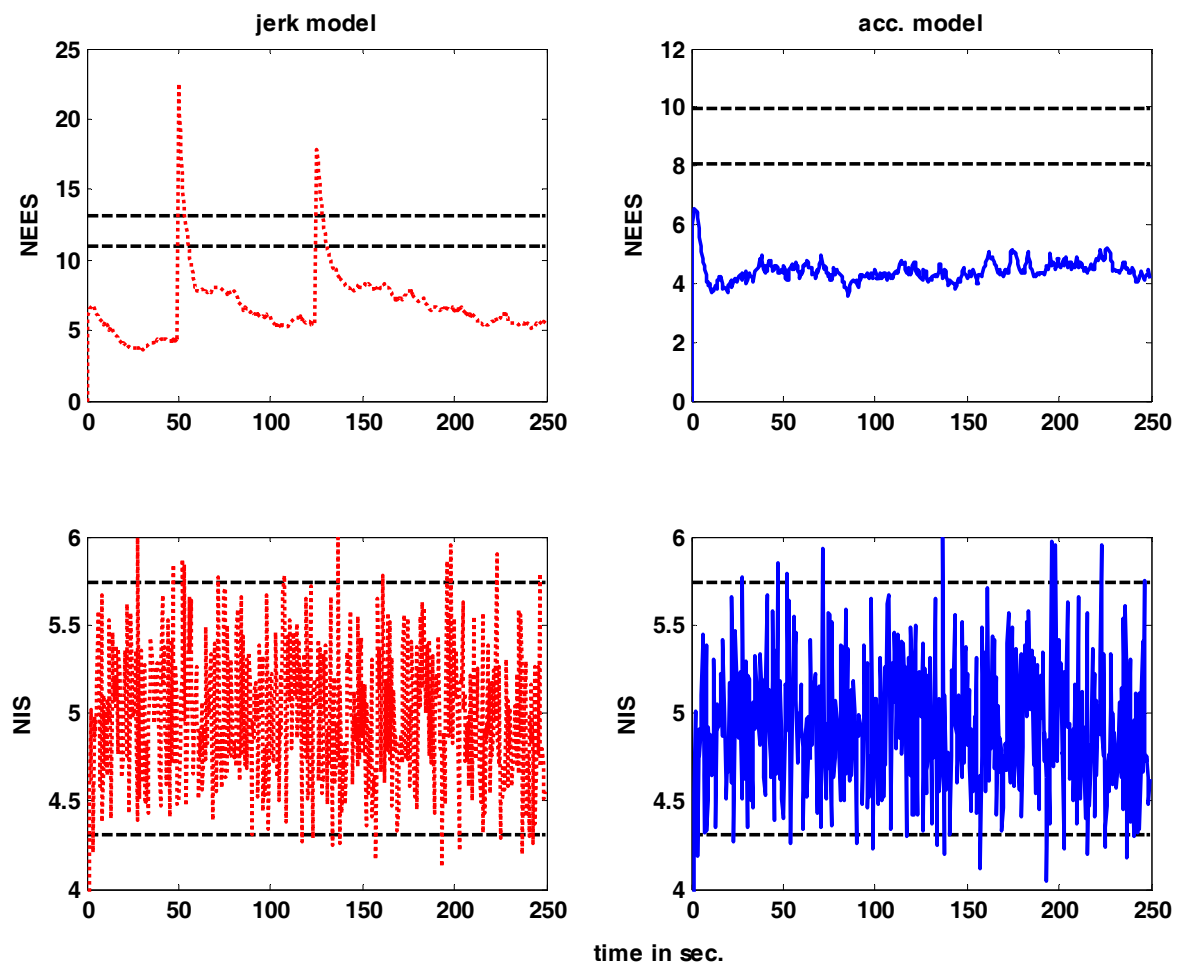

Figure 16. NEES and NIS 